\title{
Application of Business Intelligence Model in Internet Medical System
}

\author{
Siqi Li \\ Zhengzhou University Administrative Engineering College
}

Keywords: Business intelligence model, Internet medical

Abstract: The development of information technology, the continuous improvement of medical standards and the increase in the number of patients have pushed medical care to the Internet era. With the rise of "big data", the business intelligence model has been applied to business management and medical fields. Based on the relevant data, this paper analyzes the application prospect of business intelligence model in the field of Internet medical technology based on the status quo, characteristics and structure of business intelligence. Combined with the case of Internet medical platform such as "Good Doctor Online”, this paper explores the problems and facts that may be caused by the business intelligence model in the application process of the Internet medical system, in order to maximize the use of information and data, and improve the management level of hospitals and platforms. Share case data to provide patients with better medical services.

\section{Introduction}

As the population ages, the environment and the diseases caused by infectious diseases appear more and more frequently, the number of people who need to see a doctor is rising. However, the uneven distribution of medical resources and the difference in medical conditions have led to inadequate hospital treatment. People are growing their needs for medical treatment. The development of information technology has promoted the improvement of the informationization level of various medical institutions, and has also pushed the medical field to the era of "Internet + health". With the gradual popularization of Internet technologies and applications, the generated data has increased significantly. How to provide data services for the Internet medical platform systematically, timely, accurately, comprehensively and intelligently, analyze patient groups, rationally allocate medical resources, and improve medical quality. An important goal of work. Business intelligence (BI) has been introduced into Internet medical management, which is of great significance for refining patient groups, providing accurate medical services, and improving medical quality.

\section{Overview of Internet Healthcare}

\subsection{Definition of Internet Healthcare}

Internet medical, that is, in the Internet environment, the application of cloud computing, big 
data, Internet of Things and other emerging technologies, cross-infiltration with traditional medical services, integration and innovation to form a new medical model, is a new application of the Internet in the medical industry, it contains Various forms of medical health services, such as online disease consultation, electronic medical records, electronic prescriptions, and remote consultations. It represents a new development direction of the medical industry, which is conducive to solving the contradiction between China's medical resource imbalance and people's increasing health care needs. It is a medical development model actively guided and supported by the Ministry of Health (Shi Jia, 2018).

\subsection{Characteristics of Internet Medical}

Internet medical has the characteristics of openness, interaction, convenience and cross-border. It is conducive to optimizing the allocation and utilization of medical resources, simplifying the medical treatment process, providing patients with more personalized and differentiated services, greatly alleviating the difficulty of "seeing a doctor". The status quo has improved the rate of diagnosis and treatment.

(1) Let patients take the initiative

Doctors who are professional, quick and meticulous are more popular with patients. The Internet allows patients to learn more about hospitals and doctors and make more informed choices.

(2)Personalized service

With the continuous advancement of technology, smart wearable devices, health data detection devices, self-test medical devices, etc. have emerged, users can detect routine physical indicators, and easily synchronize the generated data indicators to the Internet and the cloud, with hospitals, doctors Sharing, to achieve continuous monitoring of personal health, so as to give timely medical guidance.

(3) Effectively alleviating uneven distribution of medical resources

The distribution of medical resources in China is uneven, and the medical resources in first-tier cities are numerous, while the medical resources in some remote areas and counties and villages are relatively scarce. As an interactive platform, Internet medical care can realize the sharing of medical resources. The developed cities combine with the surrounding areas to help each other and improve the medical level.

(4) Facilitating more effective supervision of medical institutions by competent authorities

In the past, prescriptions and case data of hospitals were scattered in various hospitals. By setting up an Internet supervision platform, doctors' medical behaviors are presented online in real time, which is conducive to the competent authorities to curb "big prescriptions" and alleviate the "expensive medical treatment" of ordinary people. Through Internet supervision, doctors use drugs more rationally.

\subsection{The status quo of Internet medical development}

The development of science and technology has made the impact of the Internet on the whole society have entered a new stage. With the aging of China and the increase of chronic diseases, the demand for medical and health resources has also increased. The contradiction between supply and demand of medical resources has spawned the integration of Internet technology and medical industry to a certain extent. The huge Internet users have laid the foundation for the development of the Internet medical industry.

(1) Development of science and technology

With the development of Internet technology, the infrastructure of 3G and 4G networks, wireless networks, and optical fibers has been continuously upgraded, providing a channel for massive data 
transmission and interconnection between users and platforms. In addition, the development of related technologies such as cloud computing and mobile health has met the storage space required by the hospital's continuously growing patient data and other data, thus providing a technological foundation for Internet medical care.

(2) Support for national policies

In 2015, the State Council issued the "Guiding Opinions of the State Council on Actively Promoting the "Internet +" Action" and further refined the application of the Internet in the field of health care. The opinion pointed out that it is necessary to promote the new mode of online medical and health care, and support third-party organizations to build medical information supply and marketing service platforms such as health records, electronic medical records, and inspection reports, and encourage Internet companies to cooperate with medical institutions to establish medical network information platforms and make full use of the Internet and big data. And other means to improve the ability of medical services.

(3) Improvement of the industrial chain

At present, China's Internet medical industry has formed a relatively complete industrial chain, integrating a number of participants including mobile medical service providers, medical device manufacturers, mobile operators, application developers, and insurance companies. The industrial structure dominated by medical and wearable devices. In the future, the Internet medical industry chain will develop in the depth direction

\section{The application prospect of business intelligence in the field of Internet medical}

\subsection{The concept of business intelligence}

Business intelligence is often understood as a tool that transforms existing data in the enterprise into knowledge and helps companies make informed business decisions. Business Intelligence (BI) is a collection, organization, and analysis that is a set of methods and techniques for transforming raw rough data into meaningful, useful information and operability. Business intelligence is a collection of information technologies, including Data Warehouse, Online Analytical Processing, Date Mining, and Data Visualization. These technologies have been used in the medical field in recent years. Improve the operational efficiency and quality of the organization; integrate data from heterogeneous systems in medical institutions, commit to data mining, knowledge discovery, and achieve in-depth services to provide more accurate and clear data protection for hospital leadership decisions. An increasingly complex and rapidly changing medical environment (Zhang Huihui , 2014) .

\subsection{The structure of business intelligence}

From the perspective of system architecture, business intelligence systems generally consist of data warehouse, data analysis, data mining, online analysis, data backup and recovery. The business intelligence system extracts useful data from the data collected by different data sources, cleans the data to ensure the correctness of the data, converts the data, reconstructs it and stores it in the data warehouse or data field (the data becomes information at this time) ), then find the appropriate query and analysis tools, data mining tools and O LAP tools to process the information, and finally present the knowledge to the user and turn into decision-making (as shown below) (Liu Yezheng, 2004). 


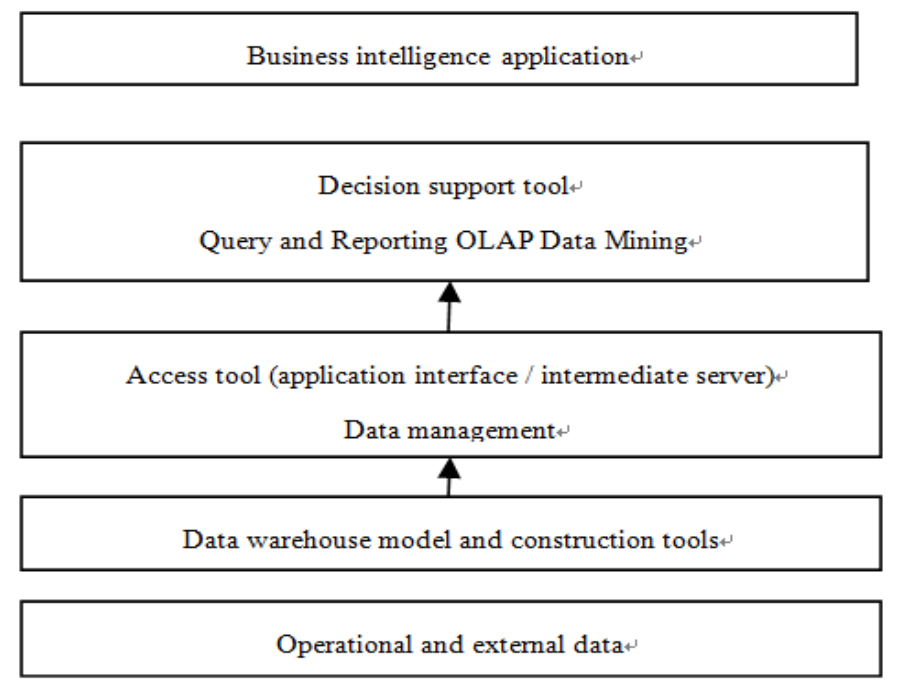

Fig.1 data source

The data source can be various data accumulated by the daily operation of the platform, or it can be external data, which is of great significance for the realization of business intelligence. One purpose of data warehousing is to transform the enterprise's information access infrastructure from an unstructured or evolving environment into a structured or well-planned environment. ETL is the core and soul of intelligent business (BI) and data mining (DW). It integrates and improves the quality of data according to unified rules. It is responsible for the process of transforming data from data source to target data warehouse. It is important to implement data warehouse. step. Data Mining uses mathematical methods such as mathematics, statistics, and artificial intelligence to mine hidden, potentially valuable relationships, patterns, and trends from large amounts of data, and use these knowledge and rules to establish decision support. Model (Dai Huan, 2017).

\subsection{The application prospect of business intelligence in the field of Internet medical}

With the advancement of medical informatization, the Internet medical platform has accumulated a large amount of management and medical data. Through business intelligence technology, we have discovered rules, predicted trends, judged primary and secondary, and early warning monitoring from these large amounts of data, thus guiding management decisions to be Internet medical. An important method of development. The core goal of business intelligence technology is to conduct comprehensive statistical calculations of business data from various angles through online analytical processing, and extract hidden and useful knowledge and information from a large amount of data (Zhang Yi,2016) .

Internet medical care is to create a platform for suppliers and users to communicate their condition and determine treatment plans. With the increasing use of platform applications, large-scale medical data, complex formats, and the lack of tools to efficiently collect, transform, and analyze data, data utilization efficiency is still low. How to integrate various information resources, carry out effective development and utilization, build a patient-centered, broad-based application field, and the data analysis platform for different information users is particularly urgent and important (Wang Zhifa, 2016) . To this end, the introduction of business intelligence technology into the field of hospital information statistics, the establishment of a BI system focusing on the integration of business data and decision-making analysis. Establishing a complete business intelligence system in the Internet medical field can systematically analyze the massive data 
generated by patients who come to see a doctor, monitor the patient's condition in real time, and make a reasonable diagnosis and adjustment through extensive analysis of changes in the patient's physical indicators.The application of BI system not only makes a reasonable analysis and efficient use of a large number of unmanageable data, but also better provides services for patients, improving management efficiency and therapeutic effect (Che Shihong, 2018) .

Take the AI intelligent consultation as an example. According to the case data of the past patients, the Good Pharmacist APP contains more than 100 kinds of common disease consultation plans and medication plans. If you feel uncomfortable, you can follow the guidance of the APP. Choose the symptoms that correspond to yourself in one step. The intelligent system will extract similar cases and treatment plans from the database, and give a medication plan or related medical advice for symptomatic treatment (Peng Chuanwei, 2014) . To a certain extent, the rational use of data has been realized, and the efficiency of medical treatment has been improved to some extent, and medical resources have been saved.

\section{Implementation countermeasures for problems in business intelligence in Internet medical treatment}

\subsection{Problems in Business Intelligence in Internet Medicine}

(1) Medical data is complicated

Due to the complex and diversified population of Internet medical services, medical data is more complicated than other fields. And medical data is often time-sensitive, requiring information to be as accurate as possible, and these are often limited by actual conditions. Internet medical data is provided by multiple application software vendors. Although each system integrates and interacts at the application level, the data is distributed and stored, and the integration is low. It is difficult to support comprehensive analysis across domains. This has made the analysis and integration of data more difficult to a certain extent, and it has made Internet medical treatment more demanding for the perfection of business intelligence systems.

(2) Lack of talents and difficulty in development

The construction of business intelligence in Internet medical care is a complex project involving all aspects of hospitals, doctors and patients. It is related to the efficient operation of hospitals and the physical and mental health of patients. It needs to fully consider the needs of patients and medical personnel, and give developers Work has increased the difficulty. Moreover, as an emerging field, Internet medical care has relatively few professional talents, and the application of business intelligence in the medical field is a new breakthrough. At the same time, new talents with medical expertise and business intelligence system technology are still scarce resources, while medical specialties. The incomplete knowledge makes it impossible for developers to fully consider the needs of patients and doctors, making development difficult. Moreover, the medical field has higher requirements for related technologies. The business intelligence system must be easy to construct and expand. It has rapid deployment and rapid response capability to meet the complex application needs of the medical and health fields and flexibly adapt to the development of the medical and health industry.

(3) Need strong financial support

The development, investment and maintenance of business intelligence, the introduction of professional talents, training, etc. all require strong financial support. This requires manufacturers to have a strong capital chain, able to provide advanced equipment, talent and technical support to ensure the continuous, steady and safe operation of the Internet medical platform.

(4) There are hidden dangers of network security

The business intelligence system is built on the computer network platform. Some system 
vulnerabilities, hacker malicious intrusions and other hidden dangers may steal or even maliciously spread the patient's medical records. In the process of being managed and utilized, due to insufficient management of medical records of hospitals and medical staff, lack of legal awareness and hidden dangers of network security, the privacy of patients is easily violated. The Internet medical information construction is the maximum information sharing under the premise of information security. Therefore, it is especially important to protect the patient's condition and privacy from the system (Zhu Jiaofeng, 2017).

(5) Powers and responsibilities are not clear

According to the main body of Internet medical treatment, China's Internet medical construction can be divided into four types: government-led multi-participation model, hospital self-construction model, company self-construction model, hospital and company joint development model. The chaos of the construction subject will lead to unclear powers and responsibilities, and the use of new medical technologies has caused some confusion in the normal medical order, which requires in-depth study in medical, legal and ethical aspects. At present, telemedicine has crossed the geographical restrictions and broke through the traditional medical model. At the same time, it has brought many new medical disputes. In medical malpractice disputes, the identification of accident liability has become a top priority. The security of data information, the accuracy of data transmission, the legal effect of electronic medical records, and the division of legal responsibilities have become important issues to consider.

\subsection{Implementation Countermeasures of Business Intelligence in Internet Medical}

Because the medical industry is related to human health and life safety, and the implementation of information technology is often accompanied by expensive investment, the attitude of medical institutions to information technology is often cautious and sensitive. The business intelligence system is a complex system. Its implementation depends not only on technical factors, but also economic, medical environment, personnel, organization and other factors. Therefore, implementing business intelligence in the medical field is not a one-step process and requires preparation and efforts from all sides.

\subsubsection{Strengthen the awareness and attention of relevant personnel}

Business intelligence is not just an information management system that performs system analysis with relatively large data. It is an advanced management concept and means. Major Internet platforms, medical application software vendors, medical personnel and other relevant personnel should fully recognize the role of business intelligence in Internet medical care and raise awareness and attention to the application of intelligent systems. At the same time, actively learn advanced experience, strengthen the training of technicians and operators, and make them realize the value of the generated big data, so as to actively carry out data mining and analysis to ensure the smooth development of medical business intelligence.

\subsubsection{Strengthening the infrastructure of information technology}

At present, although the informationization process is accelerating in China, the infrastructure is still weak, and there are problems such as incomplete data and insufficient standards. However, medical informatization is the foundation and necessary condition of business intelligence. Only by relying on the support of information technology can business intelligence develop. To make the right decision is inseparable from reliable and accurate data. Therefore, if you want to apply the business intelligence model to the Internet medical field perfectly, you must strengthen information infrastructure, improve the medical information system, and strengthen the accuracy of Internet 
medical data. Standardized control, strengthen the integration of business intelligence systems and other systems, and give full play to the role of business intelligence in medical management, big data analysis and providing personalized medical services.

\subsubsection{Talent, capital and technical preparation}

Business intelligence in the medical field is an emerging market, and domestic experienced manufacturers and related technical personnel are seriously lacking. Relevant vendors and platform owners should pay attention to the introduction and cultivation of talents. Select outstanding talents from major college graduates and social and technical personnel, and develop a series of training programs to improve the knowledge and ability of technicians to apply to Internet medical and business intelligence systems, and learn the advanced models of Internet medical and business intelligence models at home and abroad. Experience, learn from each other's strengths, and train a group of comprehensive talents who have relevant information and technology. At the same time, the platform must ensure sufficient funds and formulate a special fund plan to ensure that the business intelligence system has strong financial support from all aspects of development, operation and maintenance to ensure the safe and steady operation of the system.

\subsubsection{Strengthening network information security supervision}

Establish and improve the network security and information management system. The Internet medical platforms at all levels shall formulate relevant rules and regulations in accordance with the laws and regulations related to network and information security, clarify various responsibilities in the work, and standardize the internal control and management of the Internet platform and business intelligence systems. System, do a good job in security. Strengthen the firewall construction of Internet medical platform and business intelligence system, effectively prevent hacker attacks and information leakage, improve the security awareness of Internet use, strengthen technical protection measures such as identity authentication, access control, security audit, etc., and effectively supervise illegal operations. Conduct network and information security checks on a regular basis.

\subsubsection{Improvement of laws and regulations}

There are three roles in the application of Internet medical activities and business intelligence systems; local medical parties, Internet medical parties, and patients. Then there are three kinds of relationships between the three parties. In addition, as an indispensable part of the Internet medical activities, the network platform must also be regulated by laws to clearly define the responsibility and authority, so as to ensure Internet medical treatment. The activity was carried out smoothly and safely (Wang Miye, 2012).

\section{The enlightenment brought by "good doctor online"}

Take the Internet medical "Good Doctor Online” jointly developed by doctors and companies as an example. It establishes the first real-time updated outpatient information inquiry system on the Internet, integrating inquiry, consultation, appointment, sharing and other functions. It is China's leading Medical information and doctor-patient interaction platform. Its operating mode is mainly B2B2C mode, which forms a supply chain system between hospitals, service providers and users, breaking the $\mathrm{B} 2 \mathrm{C}$ mode of traditional hospitals and users. To a certain extent, it solves the problem of inconvenient hospital visits, and at the same time, it can conduct long-term real-time monitoring of chronic patients and improve the quality of medical care. 


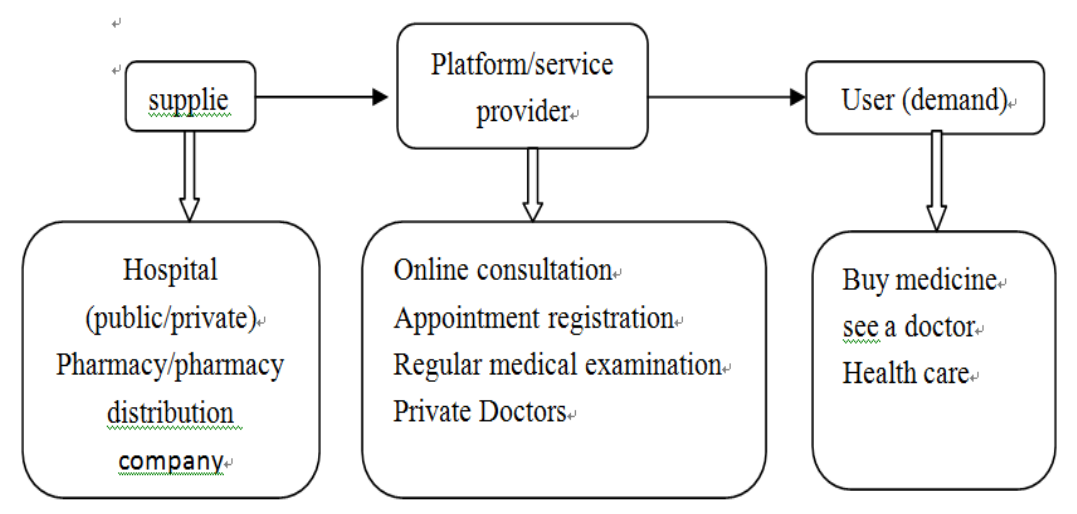

Fig.2 Platform structure

“Good Doctor Online” has created a huge information exchange platform covering hospitals, doctors and patients. It analyzes a huge amount of information including many hospitals, doctors and patients in the country, and recommends patients according to their oral conditions and medical records. Appropriate hospitals and professional doctors are available for patients to choose. With the increasing number of online consultations, the introduction of a business intelligence system can analyze a large number of cases and large amounts of data generated, and analyze the patient's condition from the aspects of age, season, and severity of the disease. The differences in cognition, the classification and grading of patients, the adoption of different regulatory measures and corresponding treatment programs can improve medical efficiency, increase experience, and bring a more comfortable treatment experience to patients.

However, there are still many drawbacks to online consultation through Internet medical platforms such as "Good Doctor Online". For example, the patient's condition mainly depends on the patient's complaint, and it is difficult for doctors to visually observe the patient's hidden symptoms through the computer, thus leading to making The diagnosis is not accurate enough. These shortcomings still need to be taken seriously, and they are constantly overcome in the operation process by continuously improving the system and strengthening the system construction.

\section{Conclusion}

In summary, this paper analyzes and studies the application of business intelligence model in the Internet medical system, and has achieved certain results. Based on the status quo of Internet medical care and the structure of business intelligence, this paper analyzes the prospects, possible problems and corresponding implementation strategies of business intelligence models in the Internet medical system. Combined with the example of “Good Doctor Online" and other leading developments in the Internet medical field, it is proposed that the implementation of the business intelligence model in the Internet medical system should pay attention to the following points: strengthen the understanding and attention of relevant personnel, and strengthen the information infrastructure. Ensure data quality, adequate preparation of talents, capital and technology, strengthen supervision of network information security, and improve laws and regulations. In the process of practice, we constantly improve the technical level, constantly improve the business intelligence model, make full use of big data information analysis technology, improve the precision of medical treatment and hospital management efficiency, and serve the majority of patients. 


\section{References}

[1] Chen Hongjun. Effective Decision of Business Intelligence Based on Big Data [J]. Enterprise Management, 2018(04): 101-103.

[2] Che Shihong. The role of business intelligence in hospital management [J]. Today Technology, 2018 (03): 59-60.

[3] Dai Huan. Design and Implementation of Data Analysis System Based on Business Intelligence [J]. Science and Technology Plaza, 2017 (02): 52-56.

[4] Liu Yezheng, Hu Jian. Research on the core technology and architecture of business intelligence [J]. Journal of Hefei University of Technology (Natural Science), 2004(08): 882-885.

[5] Peng Chuanwei, Zhong Yinli, Liu Wei. Application of hospital decision support system based on business intelligence [J]. Hospital Management, 2014, 34(08): 36-39.

[6] Shi Jia. The application of big data and business intelligence in the era of cloud consumption [J]. China market 2018 (11): 187-189.

[7] Wang Zhifa, Zhang Guiying, Lu Zhanfeng, Qiao Xiangdong, Zhang Xu. Application of Business Intelligence in Hospital Information Construction [J]. China Health Quality Management, 2016, 23(06): 73-76.

[8] Wang Miye, Huang Yong, Bi Yongdong, Zheng Tao, Zhang Rui. Application of Hospital Business Intelligence System [J]. Medical and Health Equipment, 2012, 33(01): 82-84.

[9] Zhu Jiaofeng, Ma Dong, Zheng Xiaping, Luo Bin, Dong Siping. Construction of medical risk early warning assessment model based on business intelligence technology [J]. China Health Quality Management, 2017, 24(01): 4-6. [10] Zhang Yi, Li Ke. Design and implementation of management information decision platform based on business intelligence hospital [J].China Medical Equipment, 2016, 13(11):107-110.

[11] Zhang Huihui, Ma Jingdong, Pei Jinping. Application of Business Intelligence in Health Care [J]. Chinese Journal of Health Information Management, 2014, 11 (03): 255-259. 